Micellization and adsorption of heterogemini surfactantscontaining a hydroxyl head group in aqueous solution

Tianhua Zhou, $*^{*+,}{ }^{\ddagger}$ Shengwei Liu, ${ }^{*}$ Yi You, ${ }^{\dagger}$ Rong Xu, ${ }^{\ddagger}$ and Jianxi Zhao*,+

${ }^{a}$ College of Chemistry, Fuzhou University, Fuzhou, 350002, People's Republic of China

${ }^{b}$ School of Chemical \& Biomedical Engineering, Nanyang Technological University, 62 Nanyang Drive, Singapore 637459 


\section{Synthesis of $\mathrm{C}_{m} \mathrm{OhpNC}_{\mathrm{n}}$.}

Heterogemini surfactants $\mathrm{C}_{\mathrm{m}} \mathrm{OhpNC} \mathrm{n}_{\mathrm{n}}$ were synthesized according to the reported method. ${ }^{1} \mathrm{~A}$ typical procedure was shown as below. A mixture of ethanol $(48 \mathrm{~mL})$, water $(12 \mathrm{~mL}), \mathrm{N}, \mathrm{N}^{\prime}$-dimethylalkyl amine $(0.01 \mathrm{mmol}, 1.0$ equivalent) and $\mathrm{N}, \mathrm{N}$ '-dimethylalkyl amine hydrobromide $(0.01 \mathrm{mmol}, 1.0$ equivalent) was heated to $50{ }^{\circ} \mathrm{C}$ under stirring until complete dissolution. Then, alkyl glycidyl ether (0.01 mmol, 1.0 equivalent) prepared from epichlorohydrin according literature procedure ${ }^{2}$ was added under vigorous agitation. After reaction for $4 \mathrm{~h}$, the mixture was cooled down to room temperature and the solvent was removed in a vacuum. The resultant product was recrystallized from ethyl acetate for three times, followed by vacuum drying to give compounds of the general formula $\mathrm{C}_{\mathrm{m}} \mathrm{OhpNC}_{\mathrm{n}}(\mathrm{m}, \mathrm{n}=10,8 ; 12,8 ; 14,8 ; 16,8$; and 10,14) in 33-81\% overall yield as a white solid. No attempt was made to optimize yield.

Table S1. Sample description Table

\begin{tabular}{|c|c|c|c|c|c|}
\hline Chemical & Source & $\begin{array}{l}\text { Purity } \\
\text { fraction) }\end{array}$ & (mass & Purification method & Analysis method \\
\hline $\mathrm{C}_{\mathrm{m}} \mathrm{OhpNC}_{\mathrm{n}}$ & Synthesis & $>0.99$ & & recrystallization & $\begin{array}{l}\text { Elemental analysis } \\
\text { And }{ }^{1} \mathrm{H} \text { NMR }\end{array}$ \\
\hline Ethanol & Sinopharm & $>0.99$ & & commercial & commercial \\
\hline $\mathrm{CPC}^{\mathrm{a}}$ & Sinopharm & $>0.99$ & & commercial & commercial \\
\hline Acetone & Sinopharm & $>0.99$ & & commercial & commercial \\
\hline \multicolumn{6}{|c|}{${ }^{a}$ Cetylpyridinium chloride } \\
\hline
\end{tabular}


Table S2. Semilogarithmic Data of Surface Tension $(\gamma)$ against the molality $(b)$ of at pressure $0.1 \mathrm{MPa}^{a}$ and temperature $25.0^{\circ} \mathrm{C}^{a}$.

\begin{tabular}{|c|c|c|c|c|c|c|c|c|c|}
\hline \multicolumn{2}{|c|}{$\mathrm{C}_{10} \mathrm{OhpNC}_{8}$} & \multicolumn{2}{|c|}{$\mathrm{C}_{12} \mathrm{OhpNC}_{8}$} & \multicolumn{2}{|c|}{$\mathrm{C}_{14} \mathrm{OhpNC}_{8}$} & \multicolumn{2}{|c|}{$\mathrm{C}_{16} \mathrm{OhpNC}_{8}$} & \multicolumn{2}{|c|}{$\mathrm{C}_{10} \mathrm{OhpNC}_{14}$} \\
\hline 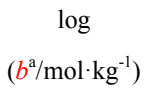 & $\gamma\left(\mathrm{mN} \cdot \mathrm{m}^{-1}\right)$ & $\begin{array}{c}\log \\
\left(b / \mathrm{mol}^{\prime} \mathrm{kg}^{-1}\right)\end{array}$ & $\begin{array}{c}\gamma \\
\left(\mathrm{mN} \cdot \mathrm{m}^{-1}\right)\end{array}$ & $\begin{array}{c}\log \\
\left(b / \mathrm{mol} \cdot \mathrm{kg}^{-1}\right)\end{array}$ & $\begin{array}{c}\gamma \\
\left(\mathrm{mN} \cdot \mathrm{m}^{-1}\right)\end{array}$ & $\begin{array}{c}\log \\
\left(b / \mathrm{mol}^{\prime} \mathrm{kg}^{-1}\right)\end{array}$ & $\begin{array}{c}\gamma \\
\left(\mathrm{mN} \cdot \mathrm{m}^{-1}\right)\end{array}$ & $\begin{array}{c}\log \\
\left(b / \mathrm{mol} \cdot \mathrm{kg}^{-1}\right)\end{array}$ & $\begin{array}{c}\gamma \\
\left(\mathrm{mN} \cdot \mathrm{m}^{-1}\right)\end{array}$ \\
\hline-2.52 & 29.06 & -3.25 & 31.90 & -3.87 & 32.76 & -4.37 & 33.03 & -4.64 & 26.44 \\
\hline-2.67 & 29.30 & -3.38 & 32.27 & -3.99 & 32.67 & -4.53 & 32.85 & -4.82 & 27.39 \\
\hline-2.82 & 29.67 & -3.49 & 32.38 & -4.11 & 32.83 & -4.63 & 32.39 & -5.00 & 28.30 \\
\hline-2.96 & 29.95 & -3.61 & 32.58 & -4.23 & 32.60 & -4.75 & 33.16 & -5.18 & 29.36 \\
\hline-3.13 & 33.29 & -3.72 & 33.42 & -4.34 & 33.74 & -4.87 & 32.73 & -5.37 & 29.56 \\
\hline-3.28 & 35.86 & -3.83 & 36.43 & -4.47 & 36.16 & -4.99 & 37.64 & -5.55 & 33.63 \\
\hline-3.43 & 39.03 & -3.98 & 39.79 & -4.60 & 39.09 & -5.10 & 41.60 & -5.64 & 35.65 \\
\hline-3.58 & 41.95 & -4.07 & 42.50 & -4.70 & 41.50 & -5.23 & 44.65 & -5.77 & 40.95 \\
\hline-3.72 & 45.13 & -4.20 & 46.10 & -4.82 & 44.86 & -5.36 & 48.71 & -5.85 & 44.06 \\
\hline-3.82 & 47.00 & -4.31 & 48.46 & -4.93 & 47.90 & -5.39 & 55.95 & -5.94 & 48.14 \\
\hline-3.95 & 49.75 & -4.45 & 52.04 & -5.07 & 51.91 & -5.42 & 60.95 & -6.07 & 53.77 \\
\hline-4.13 & 53.71 & -4.55 & 53.97 & -5.17 & 54.53 & -5.45 & 61.94 & -6.14 & 57.18 \\
\hline-4.43 & 59.62 & -4.68 & 57.65 & -5.30 & 58.67 & -5.58 & 68.55 & -6.25 & 65.68 \\
\hline-4.47 & 60.78 & -4.76 & 60.07 & -5.38 & 59.07 & -5.69 & 69.09 & -6.38 & 69.41 \\
\hline-4.62 & 63.84 & -4.98 & 64.92 & -5.47 & 60.85 & & & -6.55 & 69.87 \\
\hline & & & & & & & & -6.62 & 70.44 \\
\hline
\end{tabular}

${ }^{a}$ The solvent is water. The standard uncertainties $u(\gamma)=0.1 \mathrm{mN} \cdot \mathrm{m}^{-1} ; u(\mathrm{~T})=0.1^{\circ} \mathrm{C} ; u(\mathrm{P})=0.01 \mathrm{MPa} ; u(b)=0.02 \mathrm{~mol} \cdot \mathrm{kg}^{-1}$. 
Table S3. $I_{1} / I_{3}$ and $I_{\mathrm{E}} / I_{\mathrm{M}}$ versus $\log b$ ( $b$ is the molality) for $\mathrm{C}_{\mathrm{m}} \mathrm{OhpNC} \mathrm{C}_{\mathrm{n}}$ in aqueous solution using pyrene as the probe at pressure $0.1 \mathrm{MPa}^{a}$ and temperature $25.0{ }^{\circ} \mathrm{C}^{a}$

\begin{tabular}{|c|c|c|c|c|c|c|c|c|c|c|c|c|c|c|}
\hline \multicolumn{3}{|c|}{$\mathrm{C}_{10} \mathrm{OhpNC}_{8}$} & \multicolumn{3}{|c|}{$\mathrm{C}_{12} \mathrm{OhpNC}_{8}$} & \multicolumn{3}{|c|}{$\mathrm{C}_{14} \mathrm{OhpNC}_{8}$} & \multicolumn{3}{|c|}{$\mathrm{C}_{16} \mathrm{OhpNC}_{8}$} & \multicolumn{3}{|c|}{$\mathrm{C}_{10} \mathrm{OhpNC}_{14}$} \\
\hline $\begin{array}{c}\log \\
\left(b / \mathrm{mol}^{\prime} \mathrm{kg}^{-1}\right)\end{array}$ & $\mathrm{I}_{1} / \mathrm{I}_{3}$ & $\mathrm{I}_{\mathrm{E}} / \mathrm{I}_{\mathrm{M}}$ & $\begin{array}{c}\log \\
\left(b / \mathrm{mol}^{\prime} \mathrm{kg}^{-1)}\right.\end{array}$ & $\mathrm{I}_{1} / \mathrm{I}_{3}$ & $\mathrm{I}_{\mathrm{E}} / \mathrm{I}_{\mathrm{M}}$ & $\begin{array}{c}\log \\
\left(b / \mathrm{mol}^{\prime} \mathrm{kg}^{-1)}\right.\end{array}$ & $\mathrm{I}_{1} / \mathrm{I}_{3}$ & $\mathrm{I}_{\mathrm{E}} / \mathrm{I}_{\mathrm{M}}$ & $\begin{array}{c}\log \\
\left(b / \mathrm{mol} \cdot \mathrm{kg}^{-1)}\right.\end{array}$ & $\mathrm{I}_{1} / \mathrm{I}_{3}$ & $\mathrm{I}_{\mathrm{E}} / \mathrm{I}_{\mathrm{M}}$ & $\begin{array}{c}\log \\
\left(b / \mathrm{mol}^{\prime} \mathrm{kg}^{-1)}\right.\end{array}$ & $\mathrm{I}_{1} / \mathrm{I}_{3}$ & $\mathrm{I}_{\mathrm{E}} / \mathrm{I}_{\mathrm{M}}$ \\
\hline-2.008 & 1.229 & 0.022 & -2.677 & 1.249 & 0.054 & -3.324 & 1.267 & 0.213 & -3.924 & 1.333 & 0.335 & -3.967 & 1.337 & 0.376 \\
\hline-2.163 & 1.240 & 0.026 & -2.831 & 1.263 & 0.071 & -3.479 & 1.259 & 0.257 & -4.021 & 1.373 & 0.392 & -4.101 & 1.388 & 0.372 \\
\hline-2.184 & 1.293 & 0.032 & -2.977 & 1.271 & 0.094 & -3.624 & 1.289 & 0.351 & -4.123 & 1.416 & 0.377 & -4.213 & 1.445 & 0.358 \\
\hline-2.443 & 1.268 & 0.039 & -3.112 & 1.339 & 0.130 & -3.760 & 1.440 & 0.452 & -4.224 & 1.491 & 0.367 & -4.365 & 1.538 & 0.333 \\
\hline-2.582 & 1.270 & 0.062 & -3.251 & 1.356 & 0.195 & -3.898 & 1.546 & 0.494 & -4.322 & 1.536 & 0.290 & -4.444 & 1.599 & 0.320 \\
\hline-2.786 & 1.310 & 0.273 & -3.455 & 1.412 & 0.304 & -4.102 & 1.613 & 0.256 & -4.402 & 1.564 & 0.229 & -4.599 & 1.647 & 0.279 \\
\hline-2.883 & 1.364 & 0.138 & -3.552 & 1.622 & 0.152 & -4.199 & 1.731 & 0.132 & -4.524 & 1.648 & 0.199 & -4.747 & 1.695 & 0.259 \\
\hline-3.008 & 1.755 & 0.049 & -3.677 & 1.779 & 0.018 & -4.324 & 1.767 & 0.062 & -4.624 & 1.682 & 0.114 & -4.842 & 1.720 & 0.215 \\
\hline-3.184 & 1.775 & 0.013 & -3.853 & 1.777 & 0.013 & -4.500 & 1.782 & 0.022 & -4.703 & 1.710 & 0.104 & -4.967 & 1.759 & 0.173 \\
\hline-3.309 & 1.780 & 0.012 & -3.978 & 1.785 & 0.013 & -4.801 & 1.787 & 0.016 & -4.924 & 1.748 & 0.077 & -5.137 & 1.767 & 0.055 \\
\hline-3.508 & 1.783 & 0.013 & -4.177 & 1.789 & 0.013 & -4.824 & 1.783 & 0.012 & -5.086 & 1.782 & 0.038 & -5.267 & 1.772 & 0.025 \\
\hline-3.658 & 1.785 & 0.011 & -4.327 & 1.787 & 0.012 & -4.974 & 1.775 & 0.017 & -5.071 & 1.782 & 0.036 & -5.444 & 1.756 & 0.021 \\
\hline-3.808 & 1.788 & 0.012 & -4.477 & 1.775 & 0.012 & -5.124 & 1.801 & 0.012 & -5.310 & 1.790 & 0.007 & -5.527 & 1.771 & 0.012 \\
\hline-3.958 & 1.784 & 0.011 & -4.627 & 1.788 & 0.012 & -5.274 & 1.789 & 0.011 & -5.413 & 1.788 & 0.005 & -5.657 & 1.790 & 0.010 \\
\hline-4.108 & 1.788 & 0.011 & -4.777 & 1.776 & 0.013 & & & & -5.410 & 1.779 & 0.012 & -5.787 & 1.774 & 0.010 \\
\hline
\end{tabular}


Table S4. The plots of specific conductivity versus the molality (b) for $\mathrm{C}_{\mathrm{m}} \mathrm{OhpNC} \mathrm{C}_{\mathrm{n}}$ aqueous solutions at pressure $0.1 \mathrm{MPa}^{a}$ and temperature $25.0^{\circ} \mathrm{C}^{a}$.

\begin{tabular}{|c|c|c|c|c|c|c|c|c|c|}
\hline \multicolumn{2}{|c|}{$\mathrm{C}_{10} \mathrm{OhpNC}_{8}$} & \multicolumn{2}{|c|}{$\mathrm{C}_{12} \mathrm{OhpNC}_{8}$} & \multicolumn{2}{|c|}{$\mathrm{C}_{14} \mathrm{OhpNC}_{8}$} & \multicolumn{2}{|c|}{$\mathrm{C}_{16} \mathrm{OhpNC}_{8}$} & \multicolumn{2}{|c|}{$\mathrm{C}_{10} \mathrm{OhpNC}_{14}$} \\
\hline $\begin{array}{c}\boldsymbol{b} \\
\left(\mathrm{mmol} \cdot \mathrm{kg}^{-1}\right)\end{array}$ & $\begin{array}{c}\kappa \\
\left(\mu \mathrm{s} \cdot \mathrm{cm}^{-1}\right)\end{array}$ & $\begin{array}{c}\boldsymbol{b} \\
\left(\mathrm{mmol} \cdot \mathrm{kg}^{-1}\right)\end{array}$ & $\begin{array}{c}\kappa \\
\left(\mu \mathrm{s} \cdot \mathrm{cm}^{-1}\right)\end{array}$ & $\begin{array}{c}\boldsymbol{b} \\
\left(\mathrm{mmol} \cdot \mathrm{kg}^{-1}\right)\end{array}$ & $\begin{array}{c}\kappa \\
\left(\mu \mathrm{s} \cdot \mathrm{cm}^{-1}\right)\end{array}$ & $\begin{array}{c}\boldsymbol{b} \\
\left(\mathrm{mmol} \cdot \mathrm{kg}^{-1}\right)\end{array}$ & $\begin{array}{c}\kappa \\
\left(\mu \mathrm{s} \cdot \mathrm{cm}^{-1}\right)\end{array}$ & $\begin{array}{c}\boldsymbol{b} \\
\left(\mathrm{mmol} \cdot \mathrm{kg}^{-1}\right)\end{array}$ & $\begin{array}{c}\kappa \\
\left(\mu \mathrm{s} \cdot \mathrm{cm}^{-1}\right)\end{array}$ \\
\hline 3.0350 & 166.60 & 0.5607 & 43.90 & 0.0900 & 13.26 & 0.0067 & 0.56 & 0.0013 & 0.28 \\
\hline 2.5798 & 150.90 & 0.5326 & 42.70 & 0.0846 & 12.72 & 0.0076 & 0.58 & 0.0027 & 0.49 \\
\hline 2.2763 & 141.50 & 0.5046 & 41.20 & 0.0792 & 12.09 & 0.0086 & 0.64 & 0.0040 & 0.49 \\
\hline 1.8210 & 128.20 & 0.4766 & 39.10 & 0.0738 & 11.42 & 0.0095 & 0.74 & 0.0053 & 0.58 \\
\hline 1.5175 & 116.00 & 0.4485 & 38.00 & 0.0684 & 10.75 & 0.0105 & 0.77 & 0.0067 & 0.80 \\
\hline 1.0623 & 93.70 & 0.4205 & 36.20 & 0.0630 & 10.01 & 0.0114 & 0.78 & 0.0080 & 1.02 \\
\hline 0.9105 & 82.70 & 0.3925 & 34.30 & 0.0576 & 9.25 & 0.0124 & 0.93 & 0.0093 & 1.11 \\
\hline 0.8350 & 75.80 & 0.3644 & 33.90 & 0.0522 & 8.45 & 0.0133 & 0.96 & 0.0107 & 1.17 \\
\hline 0.7588 & 69.70 & 0.3364 & 31.00 & 0.0468 & 7.64 & 0.0143 & 1.14 & 0.0120 & 1.57 \\
\hline 0.6070 & 57.30 & 0.3084 & 29.10 & 0.0414 & 6.83 & 0.0152 & 1.11 & 0.0133 & 1.70 \\
\hline 0.5350 & 49.60 & 0.2803 & 26.80 & 0.0360 & 6.36 & 0.0162 & 1.21 & 0.0147 & 1.66 \\
\hline 0.4553 & 43.90 & 0.2523 & 24.40 & 0.0306 & 5.21 & 0.0171 & 1.32 & 0.0160 & 2.01 \\
\hline 0.3035 & 31.60 & 0.2243 & 22.60 & 0.0252 & 4.27 & 0.0181 & 1.44 & 0.0174 & 2.11 \\
\hline 0.2350 & 23.00 & 0.1962 & 19.50 & 0.0198 & 3.43 & 0.0190 & 1.47 & 0.0187 & 2.43 \\
\hline 0.1518 & 16.10 & 0.1682 & 17.30 & 0.0144 & 2.55 & 0.0209 & 1.68 & 0.0200 & 2.61 \\
\hline 0.0759 & 8.80 & 0.1402 & 14.10 & 0.0090 & 1.61 & 0.0219 & 1.76 & 0.0214 & 2.84 \\
\hline & & 0.1121 & 11.70 & & & 0.0229 & 1.90 & 0.0227 & 2.97 \\
\hline & & 0.0841 & 10.50 & & & 0.0238 & 2.01 & 0.0240 & 3.19 \\
\hline & & 0.0561 & 6.50 & & & 0.0248 & 2.12 & 0.0254 & 3.30 \\
\hline & & 0.0280 & 3.40 & & & 0.0267 & 2.31 & 0.0267 & 3.61 \\
\hline & & 0.0140 & 2.30 & & & 0.0286 & 2.53 & 0.0280 & 3.76 \\
\hline & & & & & & 0.0305 & 2.66 & 0.0294 & 3.95 \\
\hline & & & & & & 0.0324 & 2.90 & 0.0320 & 4.24 \\
\hline & & & & & & 0.0362 & 3.28 & 0.0374 & 4.70 \\
\hline & & & & & & 0.0428 & 3.80 & 0.0400 & 5.10 \\
\hline & & & & & & 0.0476 & 4.21 & 0.0427 & 5.36 \\
\hline & & & & & & & & 0.0454 & 6.12 \\
\hline & & & & & & & & 0.0481 & 6.22 \\
\hline & & & & & & & & 0.0507 & 6.50 \\
\hline & & & & & & & & 0.0534 & 6.74 \\
\hline & & & & & & & & 0.0601 & 7.46 \\
\hline & & & & & & & & 0.0667 & 8.31 \\
\hline
\end{tabular}

${ }^{a}$ The solvent is water. The standard uncertainties $u(\gamma)=0.1 \mathrm{mN} \cdot \mathrm{m}^{-1}, u_{\mathrm{r}}(\kappa)=0.05, u(\mathrm{~T})=0.1^{\circ} \mathrm{C}$, and $u(\mathrm{P})=0.01 \mathrm{MPa} ; u(b)=0.0003 \mathrm{mmol} \cdot \mathrm{kg}^{-1}$.

1. T. H. Zhou and J. X. Zhao, J. Colloid Interface Sci., 2009, 331, 476-483.

2. S. Carrà, E. Santacesaria, M. Morbidelli, P. Schwarz and C. Divo, Industrial \& Engineering Chemistry Process Design and Development, 1979, 18, 424-427. 